\title{
Comprehensive sensitivity analysis and process risk assessment of large scale pharmaceutical crystallization processes
}

\author{
Öner, Merve; Stocks, Stuart M.; Sin, Gürkan
}

Published in:

Computers \& Chemical Engineering

Link to article, DOI:

10.1016/j.compchemeng.2020.106746

Publication date:

2020

Document Version

Peer reviewed version

Link back to DTU Orbit

Citation (APA):

Öner, M., Stocks, S. M., \& Sin, G. (2020). Comprehensive sensitivity analysis and process risk assessment of large scale pharmaceutical crystallization processes. Computers \& Chemical Engineering, 135, [106746]. https://doi.org/10.1016/j.compchemeng.2020.106746

\section{General rights}

Copyright and moral rights for the publications made accessible in the public portal are retained by the authors and/or other copyright owners and it is a condition of accessing publications that users recognise and abide by the legal requirements associated with these rights.

- Users may download and print one copy of any publication from the public portal for the purpose of private study or research.

- You may not further distribute the material or use it for any profit-making activity or commercial gain

- You may freely distribute the URL identifying the publication in the public portal 


\title{
Comprehensive Sensitivity Analysis and Process Risk Assessment of Large Scale Pharmaceutical Crystallization Processes
}

\author{
Merve Öner ${ }^{1}$, Stuart M. Stocks ${ }^{2}$ and Gürkan $\operatorname{Sin}^{1 *}$ \\ ${ }^{1}$ Process and Systems Engineering Center (PROSYS), Department of Chemical and Biochemical \\ Engineering, Technical University of Denmark, DK-2800 Kgs. Lyngby, Denmark \\ ${ }^{2}$ LEO Pharma A/S, Industriparken 55, DK-2750 Ballerup, Denmark
}

\begin{abstract}
This work presents an in silico tool that supports crystallization process development and optimization studies by means of mechanistic modeling, uncertainty identification, comprehensive sensitivity analysis and a quantified process risk assessment. Kinetic model parameters and operation design parameters are considered as a source of uncertainty and variation. Monte Carlo simulations are performed to propagate input uncertainty/variation to model output in terms of process yield, mean crystal diameter and size distribution. To quantify the individual effects and importance of these parameters, global sensitivity analysis e.g. Morris Screening and variance-based decomposition, is performed. The process risk is defined as failure to reach target product specifications and its consequences for the given design space is quantified. This promising study shows, that global uncertainty and sensitivity analysis coupled with the quantification of process risk assessment is a powerful tool and should be of interest to those participating in effective and efficient crystallization process development.
\end{abstract}

Keywords: Pharmaceutical crystallization; uncertainty analysis; global sensitivity analysis; process risk assessment; scale-up; compartmental modeling

\section{Introduction}

In the pharmaceutical industries, crystallization is a fundamental unit operation during the separation and purification of intermediate compounds and active pharmaceutical ingre-

*Corresponding author (gsi@kt.dtu.dk) 
dients (APIs). Design and control of a crystallization process is a complicated task due to strict requirements of intrinsic quality attributes of the crystal products [1. In particular, crystal size distribution (CSD), morphology, purity and solid-state form have a significant impact on the physical and chemical properties of the drug substances such as dissolution rate, solubility, bio-availability and drug efficacy. They also influence the performance of following downstream processes such as filtration, drying and milling [2, 3]. For this reason, the operation conditions of the crystallization process are of critical importance, since crystal quality attributes are strongly affected and determined by the conditions, under which the crystallizer is operated [4, 2].

Conventional pharmaceutical crystallization processes are based on a recipe-based operation, in which a process variable follows a specific recipe that was submitted to regulatory authorities. Often there is a low-level control (such as temperature control) to ensure the process variable (such as process temperature) follows the recipe trajectories throughout the process [4. Recipe-based approaches fail to deliver consistent product quality in the presence of disturbances and uncertainties in the process operation and upstream. Testing the quality of the product at the end of the process and absence of any monitoring and control actions of critical solid-state quality attributes during the process are underlying reasons of batch-to-batch variations and poor product quality. Moreover, this approach also demands a high number of experiments and accordingly a high amount of resources in order to define a robust and reliable envelope for the crystallization operation [5]. In the view of these downsides, drug regulatory authorities have encouraged industries towards replacing the quality-by-testing practise with more enhanced methods such as quality-by-design (QbD) approach during drug and manufacturing development phases [4. This is only one of the various challenges that put pressure on the pharmaceutical industries including high competition with the generic market due to patent expiration of the drugs, higher cost of R\&D for new drugs and growing demand towards greener products and processes [6, 1].

Given these circumstances, now the pharmaceutical industries are seeking more effective and economic ways to increase productivity and manufacturing efficiency without compro- 
mising the product quality. To accomplish this, initiatives must be taken beginning within the area of process development under the guidance of $\mathrm{QbD}$ approach. This is also favored by the regulatory authorities. QbD approach relies on mechanistic understanding and modeling underlying principles of the process in combination with risk management tools in order to rapidly design an effective and efficient production process that delivers consistently a product meeting desired quality attributes and performance [6, 4, 7]. A process is usually regarded as well-understood when i) all critical sources of uncertainty and variability are defined and clarified, ii) variability and uncertainty are governed by the process, iii) critical quality attributes of the product and process performance can be precisely and reliably predicted over the design space of the process parameters or material attributes 8,9 .

Despite high number of mechanistic model developments, optimization and control studies, the pharmaceutical crystallization literature still lacks for a comprehensive study that applies a global uncertainty and sensitivity analysis on a crystallization process and demonstrates a straightforward pathway to quantify the process risk of not achieving target product specifications. A limited number of published studies takes the uncertainties into account during model development, process design and control of crystallization of the pharmaceutical compounds. A few studies consider the process risks, but all of them are limited to only a qualitative assessment. To illustrate, the effects of the uncertainties in the nucleation and growth kinetic parameters on the optimum operation policies such as temperature [10, 11] or agitation [12] trajectories as well as on the average crystal size [13] are studied to aid a robust design and control. Sensitivity of average crystal size with respect to uncertainties in upstream process (water content), design (cooling time and seed mass) and model (nucleation and growth) parameters are assessed [13]. A parameter estimability framework based on the combination of two methods (a sequential orthogonalization and a variance based) to calculate sensitivity indices are presented in order to assess the reliability and sufficiency of experimental data during the estimation of the model parameters for a multi-impurity multi-dimensional population balance model [14]. A qualitative risk map of (un)desired polymorphs of an API as a function of solvent composition and crystallization time are 
extracted based on automated parallel experimentation (total 80 experiments) in order to identify a safe crystallization space [15]. The effect of uncertainties in growth parameters and operation parameters on the supersaturation during a co-crystallization process is related to the risk by means of calculating the probability of polymorphic purity [16].

This study therefore is driven by the motivation of practising a "science and risk-based approach" especially during the development of the key downstream process in the pharmaceutical industries, namely crystallization. A large-scale $(218 L)$ pharmaceutical crystallization model for both batch cooling and antisolvent crystallization is developed based on a compartmentalization approach. The compartmental model is built up under the guidance of mixing simulations by means of computational fluid dynamics (CFD), which is then coupled with a population balance model in MATLAB/Simulink environment. A steady-state compartmental model is used for the batch cooling crystallization, while a novel dynamic compartmental model is developed for the antisolvent crystallization. Uncertainty analysis comprehends the assessment of the sources of uncertainty in kinetic parameters and also the variations in operation parameter design space, and their influence on several outputs such as mean crystal size, CSD and yield as the measured process performance criteria. Sensitivity analysis decomposes the total process output variance into the individual contribution of the uncertain input parameters. Parameter ranking obtained by sensitivity analysis aims to guide design and suggest the optimized operation conditions. Finally, in order to assess the process risks due to the variations in the operation design space, firstly the probabilities of not achieving the target yield and final mean crystal size are calculated. The risk is quantified by means of multiplying this likelihood of not achieving the target product specification with the consequence of this undesired event. The key novelties of the presented study are the application of global uncertainty and sensitivity analysis on the CFD based compartmental model of pharmaceutical batch cooling and antisolvent crystallization processes and presenting a methodology to quantify the process risks of failing target product specifications. 


\section{Material and Methods}

\subsection{Process Description}

\subsubsection{Crystallizer Geometry}

In this study, the hypothetical large scale crystallizer considered for the scale-up model development is a stirred tank vessel with a dished bottom from a previous work [17] and shown in Fig. 1. The geometry of an existing pilot scale crystallizer at the pilot plant facility of the Technical University of Denmark is used as a base for the vessel size, impeller type as well as for the working volume of the crystallizer. The internal diameter of the vessel, $T$ is $0.64 \mathrm{~m}$. Maximum liquid height, $H$ is $0.74 \mathrm{~m}$ and it corresponds to the liquid volume, $V$ of $218 \mathrm{~L}$. The vessel is equipped with a marine type of impeller with a diameter, $D$ of $0.30 \mathrm{~m}$ and it is located $0.27 \mathrm{~m}$ above the bottom of the vessel. Four rectangular baffles are located with $9 \mathrm{~mm}$ clearance from the vessel wall. Cooling jacket covers only sides of the vessel and has a height of $0.60 \mathrm{~m}$ with a thickness of $0.01 \mathrm{~m}$ that corresponds a volume of around 12.3 $L$ and $1.21 \mathrm{~m}^{2}$ heat exchanger area.

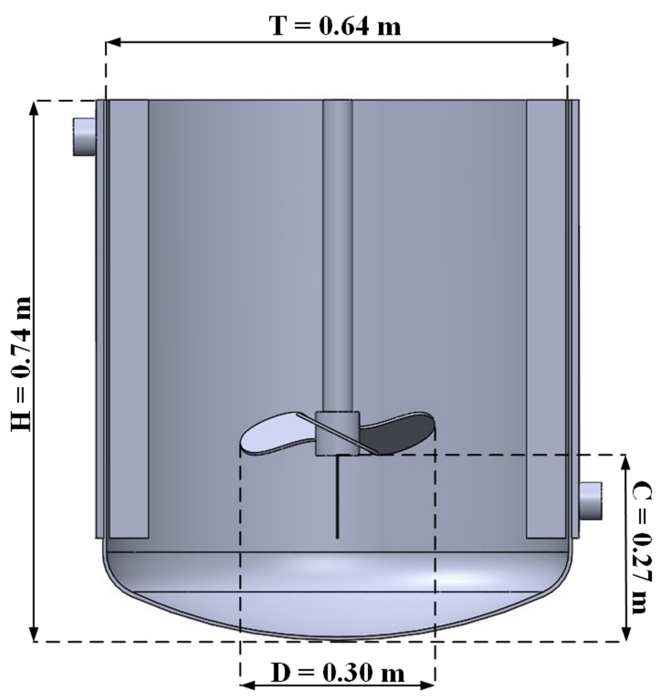

Figure 1: Pilot scale crystallizer (Adapted from previous work [17]).

\subsubsection{Crystallization Systems and Conditions}

Batch cooling crystallization: A seeded-batch cooling crystallization of an active pharmaceutical ingredient, paracetamol (Acetaminophen) from ethanol is studied as one of the case studied in this work. Related solubility and crystallization kinetic models of this 
system are taken from literature [18, 19] and given in Table 1. Operation parameters of the batch cooling crystallization process are decided as seed specifications (mass, mean, standard deviation), initial supersaturation (initial concentration), cooling profile and cooling(batch) time. Upper and lower boundaries of the design space of these operation parameters are given in Table 2. Seed is added to the solution having an initial concentration, $C_{0} \mathrm{~kg}$ paracetamol / $\mathrm{kg}$ ethanol at time, $t$ is $0 \mathrm{~min}$ and then cooling is started. The solution is cooled down from $40^{\circ} \mathrm{C}$ until $20^{\circ} \mathrm{C}$.

Table 1: Solubility and kinetic model of paracetamol crystallization from ethanol and parameter values with 95\% confidence interval.

\begin{tabular}{|c|c|c|c|c|}
\hline Name / Expression & & Parameters & & Source \\
\hline \multicolumn{2}{|l|}{ Solubility $^{a}$} & $C_{1}[k g / k g]$ & $C_{2}\left[K^{-1}\right]$ & \\
\hline \multicolumn{2}{|l|}{$C_{\text {Sat }}=C_{1} \cdot \exp \left(C_{2} \cdot T\right)$} & $2.955 \cdot 10^{-4}$ & $2.179 \cdot 10^{-2}$ & 18] \\
\hline \multirow{3}{*}{\multicolumn{2}{|c|}{ Nucleation $^{b}$}} & $k_{n}\left[\left(\# / \operatorname{minm}^{3}\right)^{n_{\text {prim }}}\right]$ & $n_{\text {Prim }}[-]$ & \\
\hline & & $(1.597 \pm 0.211) \cdot 10^{10}$ & $2.276 \pm 0.057$ & 19] \\
\hline & & $K_{n}\left[\left(\# / m^{2} s\right]\right.$ & $n_{S e c}[-]$ & \\
\hline \multicolumn{2}{|l|}{$B_{S e c}=K_{n} \cdot(\Delta c)^{n_{S e c}} \cdot \int_{L_{\min }}^{L_{\max }} n(L) \cdot L^{2} d L$} & $(2.656 \pm 0.102) \cdot 10^{7}$ & $2.232 \pm 0.086$ & 19] \\
\hline Growth $^{b}$ & $k_{g}\left[\mathrm{~m} / \mathrm{s}\left(\mathrm{m}^{3} / \mathrm{kmol}\right)^{g}\right]$ & $E_{a}[k J / m o l]$ & $g[-]$ & \\
\hline$G=k_{g} \cdot \exp \left(-\frac{E_{a}}{R \cdot T}\right) \cdot(\Delta c)^{g}$ & $9.979 \pm 0.41$ & $40.56 \pm 0.04$ & $1.602 \pm 0.03$ & 19] \\
\hline \multicolumn{5}{|c|}{$\begin{array}{l}{ }^{a} \text { Solubility is in }[\mathrm{kg} / \mathrm{kg}] \text {. Standard deviation of measured solubility data was reported as } 5.6 \cdot 10^{-4} \mathrm{~kg} / \mathrm{kg} .95 \% \\
\text { confidence interval of predicted solubility, } C_{\text {Sat }} \text { was calculated as } \pm 0.0006 \mathrm{~kg} / \mathrm{kg} \text {. }\end{array}$} \\
\hline \multicolumn{5}{|c|}{${ }^{c}$ Growth and nucleation terms are modified in order to obtain $G$ in $[\mathrm{m} / \mathrm{s}], B_{\text {Prin }}$ and $B_{S e c}$ in $\left[\# /\left(m^{3} s\right)\right]$. } \\
\hline
\end{tabular}


Table 2: Operation parameters of the batch cooling and antisolvent crystallization processes, and their design space.

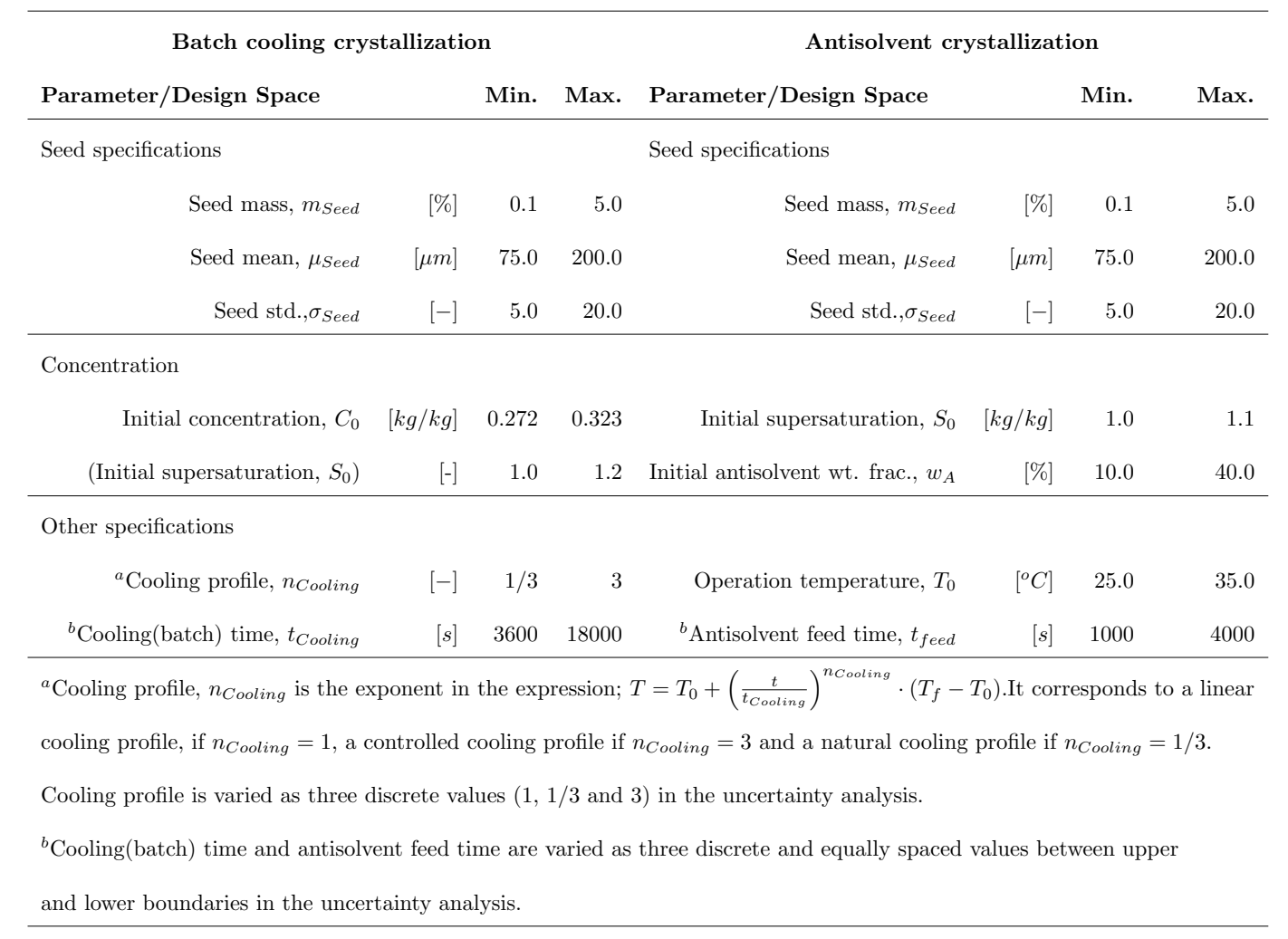

Antisolvent crystallization: Antisolvent crystallization of another active pharmaceutical ingredient, acetylsalicylic acid (Aspirin) from ethanol solvent and water antisolvent is studied as the second case in this work. Related solubility and crystallization kinetic models of this system are taken from literature [20] and given in Table 3 . Operation parameters of the antisolvent crystallization process are decided as seed specifications (mass, mean, standard deviation), initial supersaturation (initial concentration), initial antisolvent weight fraction, operation temperature and total time for addition of antisolvent (antisolvent feed time). Specifications of these operation parameters are given in Table 2 Seed is added to the solution having an initial concentration, $C_{0} \mathrm{~kg}$ acetylsalicylic acid / $\mathrm{kg}$ ethanol-water mixture at time, $t$ is $0 \mathrm{~min}$ and then antisolvent addition is started. The antisolvent is fed to the solution until final antisolvent weight fraction reaches to $60.0 \%$, which is the value studied in the literature 20$]$. 
Table 3: Solubility and kinetic model of acetylsalicylic acid crystallization from ethanol solvent and water antisolvent, and parameter values with $95 \%$ confidence interval.

\begin{tabular}{|c|c|c|c|c|}
\hline Name / Expression & \multicolumn{3}{|c|}{ Parameters } & Source \\
\hline Solubility $^{a}$ & \multicolumn{3}{|c|}{$p_{0,0}, p_{1,0}, p_{0,1}, p_{2,0}, p_{1,1}, \ldots, p_{0,6}$} & \\
\hline \multicolumn{4}{|c|}{$C_{\text {Sat }}(T, w)=p_{0,0} T^{0} w^{0}+p_{1,0} T^{1} w^{0}+p_{0,1} T^{0} w^{1}+p_{2,0} T^{2} w^{0}+p_{1,1} T^{1} w^{1}+\ldots+p_{0,6} T^{0} w^{6}$} & 20 \\
\hline Nucleation $^{b}$ & $k_{B 1}\left[m^{-3} s^{-1}\right]$ & $k_{B 2}[J / m o l]$ & $k_{B 3}[-]$ & \\
\hline$B=k_{B 1} \cdot \exp \left(-\frac{k_{B 2}}{R \cdot T}\right) \cdot \exp \left(-\frac{k_{B 3}}{l n^{2} S}\right)$ & $(1.15 \pm 0.51) \cdot 10^{21}$ & $(7.67 \pm 0.11) \cdot 10^{4}$ & $0.16 \pm 0.01$ & 20] \\
\hline Growth $^{b}$ & $k_{G 1}[\mathrm{~m} / \mathrm{s}]$ & $k_{G 2}[J / m o l]$ & $k_{G 3}[-]$ & \\
\hline$G=k_{G 1} \cdot \exp \left(-\frac{k_{G 2}}{R \cdot T}\right) \cdot\left(c^{*}(S-1)\right)^{k_{G 3}}$ & $(3.21 \pm 0.18) \cdot 10^{-4}$ & $(2.58 \pm 0.14) \cdot 10^{4}$ & $1.00 \pm 0.001$ & 20] \\
\hline \multicolumn{5}{|c|}{${ }^{a}$ Solubility is in $[\mathrm{kg} / \mathrm{kg}]$ and for parameter values please refer to the literature. } \\
\hline \multicolumn{5}{|l|}{${ }^{b} G$ in $[m / s], B$ in $\left[\# /\left(m^{3} s\right)\right]}$. \\
\hline
\end{tabular}

\subsection{Model Description}

\subsubsection{Crystallization Process Model}

Crystallization process, which is implemented in MATLAB/Simulink, is modelled as a population balance equation as shown in Eq. 1, 5, 21, and solved together with mass balance equations for solute API as well as solvents, and an energy balance equation. Method of Classes approach, which is based on discretization of internal crystal size domain into 400 classes, is used to solve the population balance equation [22, 23]. Only crystal birth (nucleation) and size-independent growth are assumed as dominant mechanisms, since only these two mechanisms are reported in the literature of the pre-mentioned API case studies. However, the developed model can easily incorporate size-dependent growth as well. Crystals are allowed to born only into the first class, $L_{0}$. Solving the model gives the time evolution of the CSD as well as all the state and process variables. In a batch system, the term $d V / d t$ becomes zero, since the volume of the suspension is constant (volume of crystals are neglected). However, in an antisolvent system, the term $d V / d t$ is dilution rate sourced from antisolvent addition.

$$
\frac{\partial n(L, t)}{\partial t}=B \delta\left(L_{0}\right)-G \frac{\partial n(L, t)}{\partial L}-\frac{n(L, t)}{V} \frac{d V}{d t}
$$




\subsubsection{CFD Model Setup}

The fluid dynamic behavior (mixing) in the crystallizer volume are predicted by performing a transient CFD simulation in ANSYS CFX release 17.1 in previous studies [17, 24. In order to simulate mixing flow of the liquid volume in a CFD software, firstly a detailed three dimensional (3D) geometry of the liquid volume inside the crystallizer vessel is defined by means of drawing it in a 3D solid modeling software e.g. SolidWorks. Afterwards, a computational mesh of the defined geometry, which consists of mesh elements and nodes, is created. ANSYS CFX utilizes a node based solver that solves gradually the flow equations for each individual flow variable (e.g. velocity, temperature) in an iterative way for each node in a simulated mesh [25]. The representative Navier-Stokes equations are solved for a single-phase volume and the standard $\mathrm{k}-\epsilon$ model is used as the turbulence model. Two rotation speeds of the impeller as 40 and $100 \mathrm{rpm}$, which produces a turbulent flow within the studied volume, are studied.

The volume of liquid flow being studied in the crystallizer vessels consists of two mesh domains: a rotating domain and a stationary domain. The rotating domain is a cylindrical mesh zone with $0.330 \mathrm{~m}$ diameter and $0.100 \mathrm{~m}$ height around the impeller rotating at the impeller speed and the stationary domain is the mesh zone covering the rest of the crystallizer. A frozen rotor approach is used between the intersection of two domains to model the rotation of the impeller. ICEM CFD 17.1 is used for meshing the domains. Structured hexahedron mesh elements form the stationary domain, while unstructured tetrahedron mesh elements are used to form the rotating domain. The time step used in the simulations is up to $0.01 \mathrm{~s}$. Several monitor points are randomly placed in the fluid volume, on which value of the flow velocity is monitored during the transient simulations. The CFD simulation is stopped, after the flow velocity at these monitor points remains constant, so the steady state behavior is obtained. Transient-average values of the flow velocity components (in axial, radial and circumferential directions) are recorded to be used for the development of the compartmental model of the system. 


\subsubsection{Compartmentalization and Modeling}

The main purpose of the compartmentalization approach is to capture the mixing behaviour particularly in large scale systems and to enable the examination of the affected process variables and state outcomes in a both cost-efficiently and accurately manner. This is achieved by defining the entire vessel as a network of interconnected ideally mixed subvolumes (compartmental zones), in which no or negligible gradients exist. Overall flow pattern, temperature distribution, concentration, local energy dissipation and solid distribution can be regarded as base criterion for determination of the compartments in crystallization vessels [26, 27, 28].

In this work, the overall flow pattern and its velocity components in the crystallizer vessel are the primary criteria utilized for the compartmentalization. CFD simulations provides a reliable insight into mixing behaviour in the large scale systems. Therefore, the flow information gained by means of CFD simulations is used to built up compartments. A compartmental map of the mixing characteristic of the fluid in the crystallizer is established through analyzing the fluid velocity in axial, radial and circumferential directions [29]. Through the compartmentalization approach, the computational cells of the CFD mesh owning to similar characteristics are gathered together to form a coarser mesh, so called compartments. Spatially averaged values of the parameters within the compartmental volume are used to represent the sub-volume's characteristics [27. Fluid flow has unidirectional characteristics on each surface of the compartments. The way that the compartments are generated and modelled allows to save the computational time, while making no/negligible concessions of the accuracy of the model [30]. A steady-state compartmental zone map is extracted for the fluid flow in a batch system. However, a novel dynamic compartmentalization approach is utilized to capture the mixing dynamics during antisolvent addition such as the changes in compartmental volumes and in-between fluxes. More details about dynamic compartmental modeling can be found both in Supplementary Material and in our previous work [24]. In the compartmental model, a single compartment exchanges flows with the neighbouring compartments (Fig, 22). Therefore, in a compartmental model, the population balance as 
well as mass and energy balance equations contain multiple inlet and outlet streams similar to continuous reactor systems that is valid for both batch and antisolvent crystallization case. Therefore, sink and source terms are added to Eq. 1 for inlet inlet and outlet streams between compartments as shown in Eq. 2.

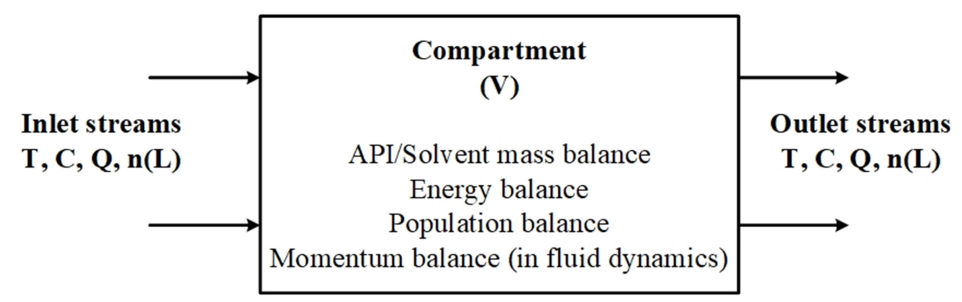

Figure 2: A single compartment model (Adapted from [28]).

$$
\frac{\partial n(L, t)}{\partial t}=B \delta\left(L_{0}\right)-G \frac{\partial n(L, t)}{\partial L}-\frac{n(L, t)}{V} \frac{d V}{d t}+\frac{n_{\text {in }}(L, t) Q_{\text {in }}-n(L, t) Q_{\text {out }}}{V}
$$

where $n$ is crystal number density, $B$ is total nucleation rate, $\delta\left(L_{0}\right)$ represents the crystal born only into the first class of $L_{0}, G$ is growth rate of the crystals, $V$ is volume, $Q$ represents the inlet and outlet volumetric flow rates. The change in solute concentration, $C$, is described as:

$$
\frac{d C}{d t}=-\frac{k_{v} \rho_{c}}{\rho_{l}} \sum L^{3} \frac{d n(L, t)}{d t}+\frac{F_{\text {in }} C_{i n}-F_{\text {out }} C}{V \rho_{l}}
$$

where $k_{v}$ is the volume shape factor of the crystals, $\rho_{c}$ is crystal density, $\rho_{l}$ is solvent density, $F$ is the inlet and outlet mass flow rates. The change in bulk temperature, $T_{b}$, and cooling water temperature, $T_{c}$ are describes as:

$$
\begin{gathered}
\rho_{l} V C_{p, l} \frac{d T_{b}}{d t}=-U A\left(T_{b}-T_{c}\right)-\Delta H_{c} \rho_{c} k_{v} \sum L^{3} \frac{d n(L, t)}{d t}+C_{p, l}\left(F_{\text {in }} T_{b, \text { in }}-F_{\text {out }} T_{b}\right) \\
\rho_{w} V_{c} C_{p, w} \frac{d T_{c}}{d t}=U A\left(T_{b}-T_{c}\right)+C_{p, w}\left(F_{\text {in }, w} T_{c, \text { in }}-F_{\text {out }, w} T_{c}\right)
\end{gathered}
$$

where $C_{p, l}$ is heat capacity of the solvent, $U$ is heat transfer coefficient, $A$ is heat transfer 
area, $\Delta H_{c}$ is heat of crystallization and $C_{p, w}$ is heat capacity of the cooling water.

\subsection{Sensitivity Analysis}

Sensitivity analysis aims to quantify and prioritize the contribution of the various sources of model uncertainties to the model output uncertainties 31. On the other hand, uncertainty analysis deals with the characterization of the sources of the uncertainty, and estimation of the uncertainty in the model prediction. Therefore it should be performed prior to sensitivity analysis [32]. A general scheme of sensitivity analysis of pharmaceutical crystallization scale-up model is represented in Fig. 3 as follows:

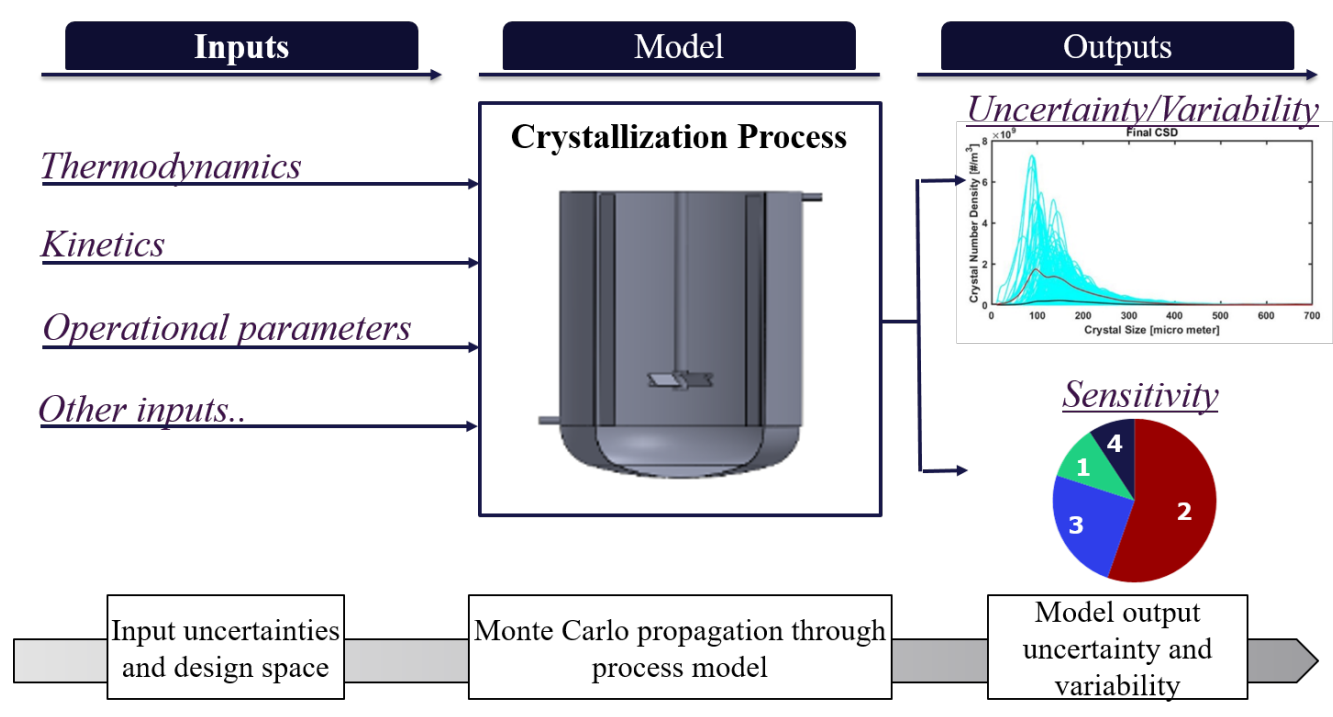

Figure 3: General overview of sensitivity analysis.

The crystallization processes and the models used to describe them can contain various sources of uncertainties such as structural uncertainty, measurement errors, parameter uncertainty and variations in the operation conditions [11. The model structural uncertainty is out of the scope of this work. The pre-mentioned crystallization process model includes various input parameters e.g. coefficients in the solubility, nucleation and growth expressions. These parameters have been determined from several experimental studies, therefore they contain experimental errors and they are often reported as a mean value with a confidence interval, which is regarded as uncertainties. Besides that, operation parameters such as initial concentration, seed mass, seed mean, seed concentration, cooling rate, etc. 
are also used in the model as input parameters. These operation parameters are process design variables that have a design space and they should be optimized. Assigning only a single value to these parameters during process model simulations will ignore all these errors. Instead, multiple values for these parameters can be defined by treating the value as a distribution within its uncertainty range. Following, multiple simulations can be performed by using these multiple assigned values for these parameters. This procedure is called Monte Carlo uncertainty propagation through the process model [33]. A probabilistic range of all the possible process outcomes due to input uncertainties will be obtained as a consequence of this procedure, called uncertainty analysis. On the other hand, in order to quantify the individual contributions of input parameter uncertainties to the total output variance, sensitivity analysis should be performed. Consequently, a parameter significance ranking is obtained and it will help to prioritize better experimental design procedures and optimization studies on those most influential parameters in order to reduce process output uncertainty and variance.

\subsubsection{Uncertainty Analysis}

Uncertainty analysis of a model system via a Monte Carlo method includes the following steps [34]:

Step 1. Definition of input uncertainty: Input (parameters) candidates, which have uncertainties, are identified and their uncertainty range as well as their distribution are defined.

Step 2. Sampling from input space: Using an appropriate sampling technique (e.g. Latin Hypercube Sampling (LHS) [35), a pre-defined number of random samples $\left(N_{\text {Sampling }}\right)$ from uncertainty range of the input parameters are created.

Step 3. Performing Monte Carlo simulations: $N_{\text {Sampling }}$ model simulations using the $N_{\text {Sampling }}$ samples of input parameters values created in the previous step, are performed.

Step 4. Reviewing and analysis of the results: The outputs of the $N_{\text {Sampling }}$ model simulations are plotted and the results are characterized into mean, standard deviation/variance and percentiles 34. 


\subsubsection{Morris Screening}

The Morris screening method is based on the calculation of the distribution of the elementary effects $\left(E E_{i}\right)$ of each input parameter by measuring the ratio of the change in model output corresponding to a change in a model input [36]. Assuming a model with $k$ independent inputs $x_{i}, i=1, \ldots, k$, each sampling run starts from a random point in a grid, where the range of each parameter space is subdivided into $p$-levels. The trajectories throughout the input parameter space are generated through perturbing one-factor-at-a-time (OAT) along a grid of size, $\Delta$ (or perturbation step) [36, 37, 38, 39. The outstanding feature of the Morris screening method is its economy in terms of required number of model simulations that is optimized by proposed OAT design by Morris [40. The degrees of freedom that are necessary to be defined are the predetermined perturbation factor, $\Delta$, a level of $p$ and the number of repetitions $r$ [37. The proposed value of $p$ is 4,6 or 8 that represents the $25^{\text {th }}, 17^{\text {th }}$ and $12.5^{\text {th }}$ percentile of the uniform distribution of the inputs. The recommended calculation for the perturbation factor is $\Delta=p /[2(p-1)]$, while the number of repetitions, $r$ takes a value typically between 10 to 50 [36, 37, 39, 41. Consequently, the random observations of the distribution function are obtained by the calculations of elementary effects as follows:

$$
E E_{i}=\frac{y\left(x_{1}, x_{2}, \ldots, x_{i-1}, x_{i}+\Delta, \ldots, x_{k}\right)-y\left(x_{1}, x_{2}, \ldots, x_{i}, \ldots, x_{k}\right)}{\Delta}
$$

where $y\left(x_{1}, x_{2}, \ldots, x_{i-1}, x_{i}, \ldots, x_{k}\right)$ is scalar model output estimated at input parameters $\left(x_{1}, x_{2}, \ldots, x_{i}, \ldots, x_{k}\right)$, while $y\left(x_{1}, x_{2}, \ldots, x_{i-1}, x_{i}+\Delta, \ldots, x_{k}\right)$ is the scalar model output regarding to a $\Delta$ change in $x_{i}$. The difference between the subsequent points and the previous is the change in one component, $i$ which has been increased or decreased. Each trajectory contains $(k+1)$ points that necessitate in total $r(k+1)$ model evaluations to get the required model output for all input samples [39. Finally, the calculated $E E_{i}$ for each input parameter trajectory can be examined by comparison of mean $(\mu)$, absolute mean $\left(\mu^{*}\right)$ and 
standard deviation $\left(\sigma^{2}\right)$ of the distribution function, which are calculated as follows:

$$
\begin{gathered}
\mu_{i}=\frac{1}{r} \sum_{j=1}^{r} E E_{i}^{j} \\
\mu_{i}^{*}=\frac{1}{r} \sum_{j=1}^{r}\left|E E_{i}^{j}\right| \\
\sigma_{i}^{2}=\frac{1}{r-1} \sum_{j=1}^{r}\left(E E_{i}^{j}-\mu_{i}\right)^{2}
\end{gathered}
$$

\subsubsection{Variance-Based Method}

Variance based sensitivity analysis method can be described as the "computer experiment equivalent" of the performance (outcome) variance analysis of an experimental design. In experimental design, the contributions of factors are studied over levels, while in variance based methods the emphasis is on entire factor distribution [42]. In the variance based sensitivity analysis method, the total variance of output has been decomposed into the fractions, which can be attributed to individual parameters and set of parameters. A direct variance-based measure of sensitivity -so called first order sensitivity index, $S_{i^{-}}$calculates the main contribution of an input parameter (by varying this parameter alone) to output variance. On the other hand, the total-order index, $S_{T i}$ measures not only the individual parameter contribution to output variance, but also all variances caused by related parameter interactions with other inputs [43, 44, 45]. Variance-based method such as Sobol's sensitivity indices employ Monte Carlo simulations, which rely on a random sampling from uncertain input space and repeated simulations [46].

\subsubsection{Meta-Modeling Based Global Sensitivity Analysis}

Variance-based methods such as Sobol's sensitivity indices require a high number of model evaluations in the order of 2,000-10,000 in order to ensure the convergence of calculated indices with a satisfying precision. Accordingly, the computational burden associated with the sensitivity analysis is very high in case of computationally expensive models are 
studied. Therefore, meta-modeling based approaches to calculate sensitivity indices have gained high interest as a cost-efficient alternative to conventional methods [46]. Polynomial chaos expansions [47, 48], Gaussian process regression [49] and artificial neural networks [50] are the most referred surrogate-models for global sensitivity analysis [46]. More details about these methods and underlying principles can be found elsewhere [46].

\subsection{Process Risk Quantification}

The steps followed to quantify process risk are shown as a schematic diagram in Fig. 4. Process risk of not achieving target crystal product specification is quantified based on the predicted probability distribution using Monte-Carlo simulations of the crystallization model.
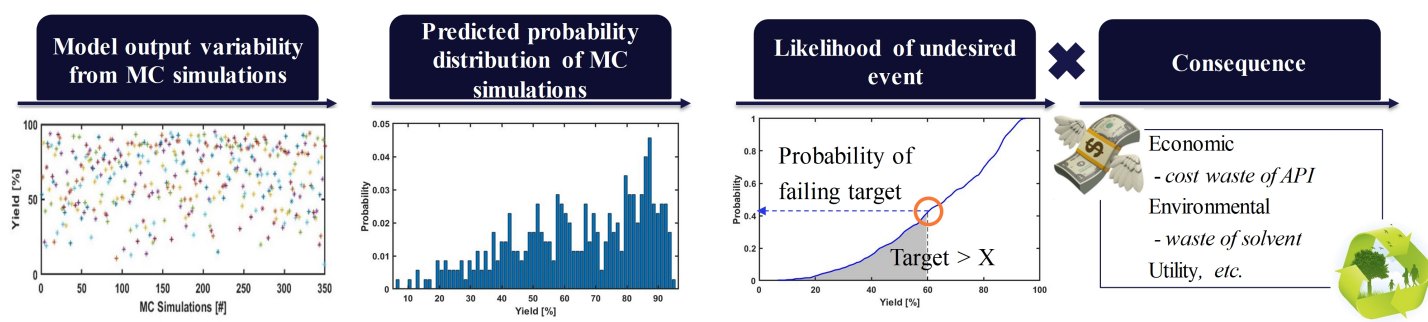

Figure 4: Schematic diagram of the steps to be followed during the quantification of the process risk based on Monte Carlo simulations.

Process risk is defined as multiplication of the likelihood of an undesired event (u.e.) with the consequence of that event [51, 52] as shown in Eq. 10 .

$$
\text { ProcessRisk }=\sum \text { Likelihood }_{\text {ofu.e. }} \cdot \text { Consequence }_{\text {ofu.e. }}
$$

Likelihood of an event (also in terms of cumulative density function) can be quantified by summing the probabilistic distribution of process outcome obtained from Monte Carlo simulations. The consequence of an undesired event can be defined as a model or function that incorporates several impact measures of this undesired event (Eq. 11) such as cost of product loss and solvent, environmental footprint of the wasted solvent etc.:

$$
\text { Consequence }=f(\text { Model })=f(\text { Economic, environmental }, \text { etc. })
$$




\section{Results and Discussion}

\subsection{CFD Simulations and Compartmentalization}

In a previous work, transient CFD simulations were performed in order to predict the fluid dynamic behavior in the crystallizer volume 24]. The developed steady state flow pattern and velocity profile in initial solvent volume $(126 \mathrm{~L})$ and in the final full-volume vessel $(218 L)$ is shown in Figure 5 . Batch cooling crystallizer is operated at the full volume of $218 L$, while in the antisolvent crystallizer, initial liquid volume of $126 L$ is increased up to $218 L$ due to antisolvent addition. CFD simulations show that one circulation loop of the liquid flow exists in the initial solvent volume, while two circulation loops of the liquid flow exist above and below the impeller in the full crystallizer volume. Liquid flow velocity has the highest value around the impeller region as expected. Steady-state compartmentalization of the full crystallizer volume can be seen also in Fig. 5. The details of the dynamic compartmentalization in order to replicate the mixing of the solvent with antisolvent and compartmental information can be found in our previous work [24].
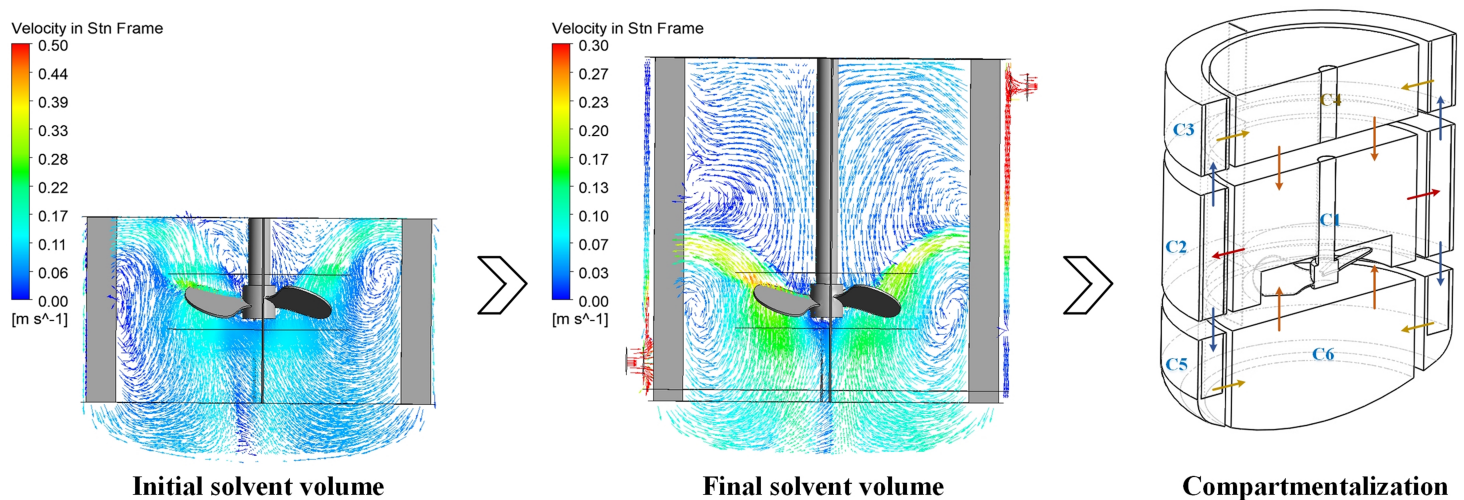

Final solvent volume

Compartmentalization

Figure 5: Fluid flow pattern in pilot scale crystallizer having initial $(126 L)$ and final $(218 L)$ liquid volumes, and compartmentalization of final liquid volume (40 rpm impeller speed).

\subsection{Case Study 1 - Batch Cooling Crystallization}

\subsubsection{Uncertainty Analysis}

Definition of uncertainty range is a crucial task. There are two methods commonly used to define an appropriate range for uncertainties in the literature; (i) If experimental data is available, parameter uncertainties are inferred using statistical estimation methods. For 
example, kinetic parameters of a mathematical model is estimated using maximum likelihood estimation (MLE) and $95 \%$ confidence interval of the kinetic parameters are used, (ii) If data is not available, the expert review is used to define an appropriate range such as low, medium and high degree of uncertainty. For example, the opinions of process experts are asked and/or related literature resources are consulted [37, 53]. The crystallization model input parameters are classified into two groups; kinetic parameters and operational parameters. During uncertainty analysis (also during sensitivity analysis) kinetic parameters and operation parameters are studied separately. The coefficients of solubility $\left(C_{1}, C_{2}\right)$, nucleation $\left(k_{n}, n_{\text {Prin }}, K_{n}, n_{\text {Sec }}\right)$ and growth terms $\left(k_{g}, g, E_{a}\right)$ (shown in Table 1 ) are regarded as uncertain kinetic parameters, that have been determined by experimental data containing errors. The uncertainty range for kinetic parameters are specified as $\pm 5 \%$ of the reported mean values. Additionally, uncertainties in volumetric shape factor, $k_{v}$, which is used in population balance modeling to calculate volume of a crystal, are also taken into account, since different reported values exist for paracetamol crystals $\left(k_{v}=0.674\right.$ [18 and $k_{v}=0.866$ [54).

On the other hand, the operation parameters such as initial solute concentration, $C_{0}$, cooling (batch) time, $t_{\text {Cooling }}$, cooling profile, $n_{\text {Cooling }}$, seed mass, $m_{\text {Seed }}$, seed standard deviation, $\sigma_{\text {Seed }}$ and seed mean, $\mu_{\text {Seed }}$ are regarded as design parameters, which should be optimized within a design space. Moreover, design space of those operation parameters poses a risk of not achieving the target product and process specifications. Therefore, this risk should be quantified. During the decision of the design space of these variables, we benefit from the experiences of some crystallization experts and the conditions studied in the literature, where the kinetic models are developed and optimized [19, 20]. The design space for the operation parameters are given in Table 2, A uniform distribution is assumed during the sampling of the parameters from their uncertainty range and design space except for cooling profile and cooling(batch) time.

350 random samples $\left(N_{\text {Sampling }}\right)$ from input uncertainty and design space for each input parameter groups are created separately with LHS technique. The created sampling 
matrices can be found in Supplementary Material. Monte Carlo simulations are performed by simulating crystallization scale-up model 350 times for both cases using created input parameter sampling matrix. While the kinetic parameters are studied, values of the operation parameters in Monte Carlo simulations are kept constant at the given values; 0.277 $\mathrm{kg} / \mathrm{kg}$ initial solute concentration (1.02 [-] initial supersaturation), $18000 \mathrm{~s}$ cooling (batch) time, controlled cooling profile, $2 \%$ seed mass with $90 \mu m$ and $10 \mu m$ standard deviation. While the operation parameters are studied, the kinetic parameters are kept constant at the reported mean values in Table 1.

Solute concentration profile, time evolution of mean crystal size, $d_{32}$ and final CSD are recorded for each simulations and shown in Fig. 6. Final yield percentage and final mean crystal size are shown in Fig. 7. Discontinuities in solute concentration profile and mean crystal size evaluation in Fig. 6 are due the fact that cooling (batch) time is varied as three discrete values between $3600-18000 \mathrm{~s}$, which is defined as the boundaries of the operation design space in previously Table 2 .
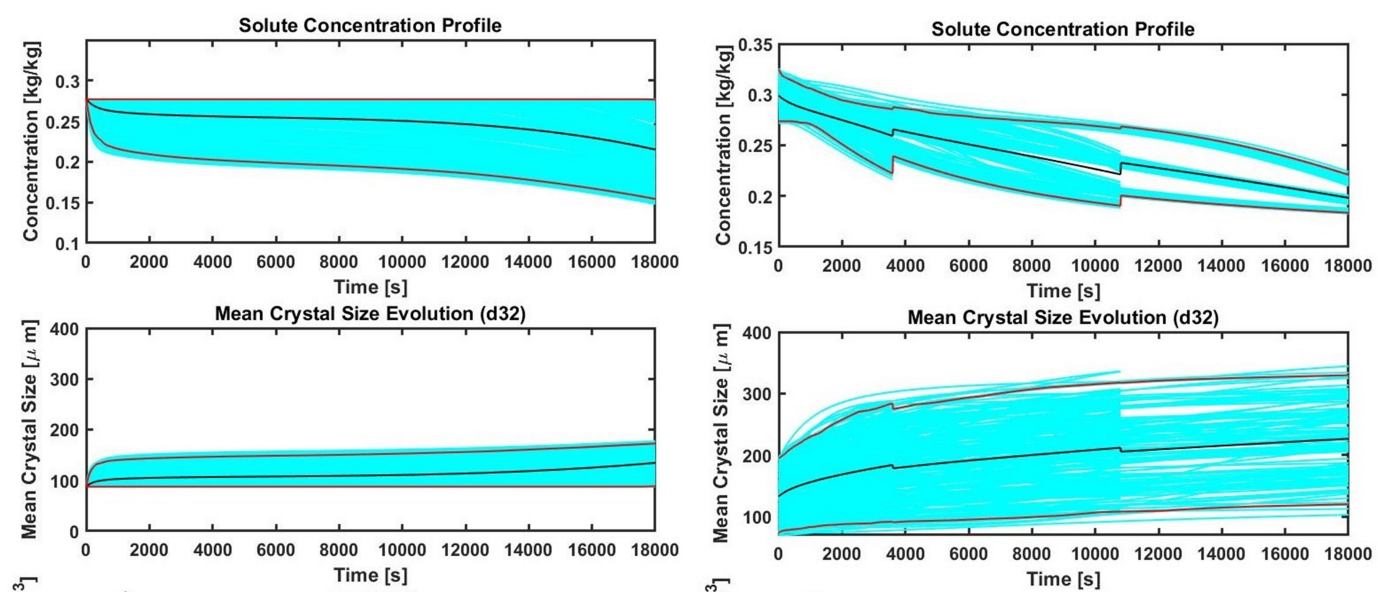

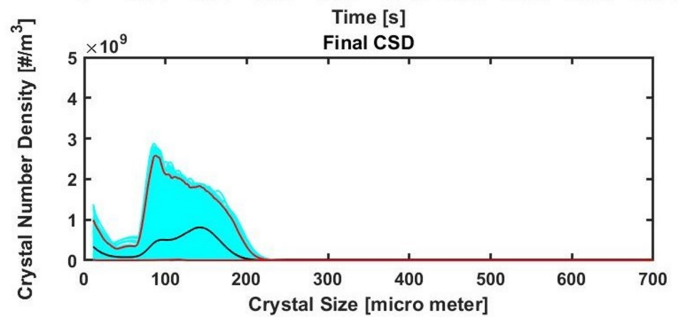

(a) Uncertainties in kinetic parameters.

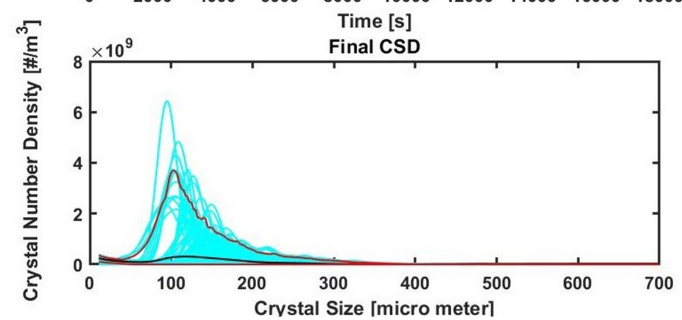

(b) Variations in operation design space.

Figure 6: $350 \mathrm{MC}$ simulations: Total output uncertainty of batch cooling crystallization due to kinetic parameter uncertainties and variations in operation design space. 

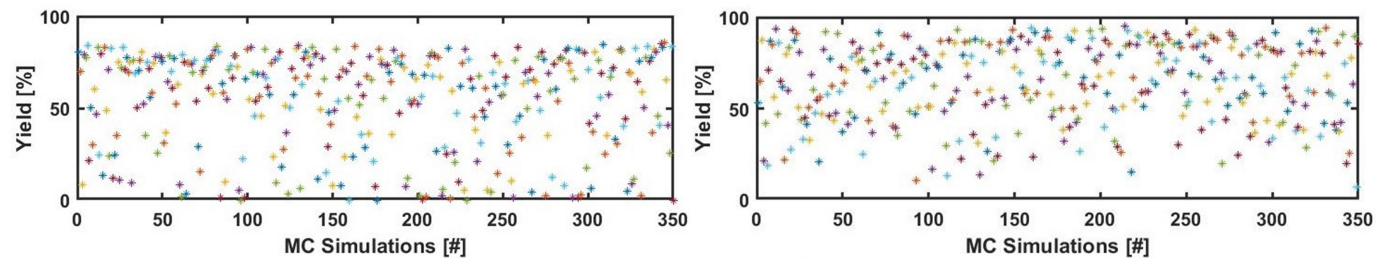

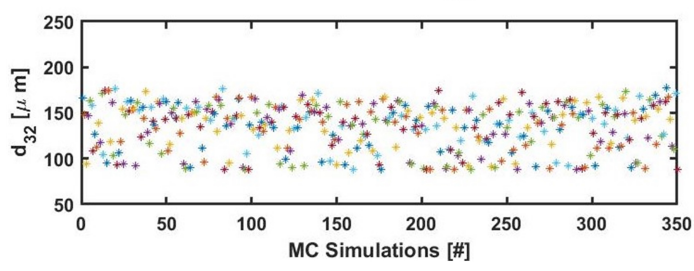

(a) Uncertainties in kinetic parameters.

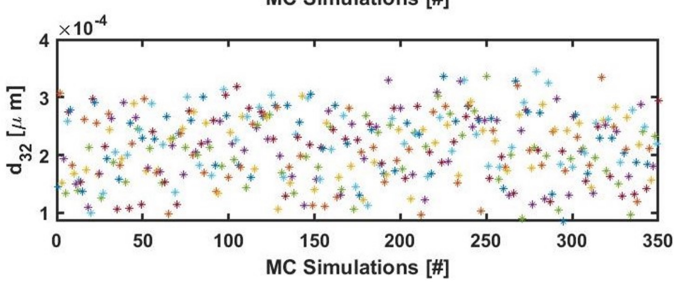

(b) Variations in operation design space.

Figure 7: 350 MC simulations: Total output uncertainty (yield and final mean crystal size) of batch cooling crystallization due to kinetic parameter uncertainties and variations in operation design space.

Fig. 6 and Fig. 7 clearly show that that $5 \%$ uncertainties in the kinetic parameters and specified variations in the operation design space lead to high uncertainties/variations in the process performance (outcome). Uncertainties in the mean crystal size at the end of the batch is much larger due to variations in the operation design space (mean of 226.6 \pm 59.3 $\mu \mathrm{m})$ compared to the uncertainties in the kinetic parameters (mean of $134.4 \pm 25.1 \mu \mathrm{m}$ ). The underlying reason can be explained as the variations of the operation parameters in specified design space have much stronger effect on the final mean crystal size compared to $5 \%$ uncertainties in the kinetic parameters. Nonetheless, the question of which operation parameter contributes the most to this huge uncertainty on the final mean crystal size will be answered by doing sensitivity analysis. Higher final average process yield is obtained in the second case with a similar standard deviation (53.9 $\pm 26.2 \%$ and $64.3 \pm 21.2 \%$, respectively).

\subsubsection{Sensitivity Analysis}

The level of $p=6$ and the perturbation factor of $\Delta=0.60$ as well as $r=20$ elementary effects calculations for each inputs are specified to design Morris sampling (Sampling matrices can be found in Supplementary Material). This results in a total of $r(k+1)=220$ model simulations for $k=10$ input kinetics parameters and $r(k+1)=140$ model simulations for $k=6$ input design parameters. The distribution of the elementary effects of each input parameters is calculated and the information about random observations of this distribution 
function is reported in terms of mean and standard deviation calculated based on Eq. 8 and Eq. 9. The significance of the model input to the model output variance is examined by comparing mean and the standard deviation of calculated distribution functions and shown in Fig. 8 for input kinetic parameters and operation parameters, respectively. In the figure, the wedge form facilitates the interpretation of the results. If an input parameter has negligible effect on the output, then this parameter lies inside of the wedge. On the contrary, if an input parameter lies outside of the wedge, it indicates that this input parameter has a significant effect on the output performance. The significance ranking list of calculated values of the absolute mean and standard deviation plotted on the Fig. 8 can be found in Supplementary Material as well.

When the sensitivity of kinetic parameters is analyzed, Morris screening method emphasizes that the second coefficient in the solubility term has the highest effect on final process yield and final crystal mean size. The highest sensitivity of the second coefficient of solubility term can be attributed to the direct correlation between this term and the temperature of system. Following, activation energy in growth expression and first solubility coefficient have also high importance. On the other hand, the coefficient of the nucleation expressions have almost zero effect on the output. These results are expected, due to the fact that under operation conditions of this crystallizer system, growth is the dominating mechanism, while nucleation is insignificant. Based on these results one should consider to focus on increasing the accuracy of these uncertain parameters, so output uncertainty can be decreased.

Sensitivity analysis of the operation parameters with respect to different outputs of interest enhances the understanding of process determinants and quantification of their contribution to process output variability. Morris screening method reveals that the crystallization process yield has strongly affected by cooling (batch) time, cooling profile and initial concentration, respectively. Seed specifications such as seed mean and mass as well as initial concentration plays the most important role in determining final mean crystal size obtained from the process. Additionally, sensitivity of the operation parameters on the span of final CSD and total nucleated crystals are also analysed (not shown on the graph). Apart from 
seed standard deviation, none of the studied operation parameter under defined space shows influence on the span of the final CSD, while initial concentration, cooling profile. cooling time and seed mass has the highest impact on the total nucleated crystals, respectively. Calculated standard deviation values of these parameters indicate that there is no interaction between these parameters and they have only additive effect on the output variance. Therefore, it can be concluded that if the aim is to optimize of process yield, seed specifications should be excluded from operation design space and focus should be given primarily to cooling time and profile. Oppositely, if the purpose is to optimize final mean crystal size, then seed specifications should be first choice of optimization. Parameter significance ranking obtained by Morris screening method are also verified by by surrogate based variance decomposition method such as PCE and ANN (Details of the results can be found in Supplementary Material).
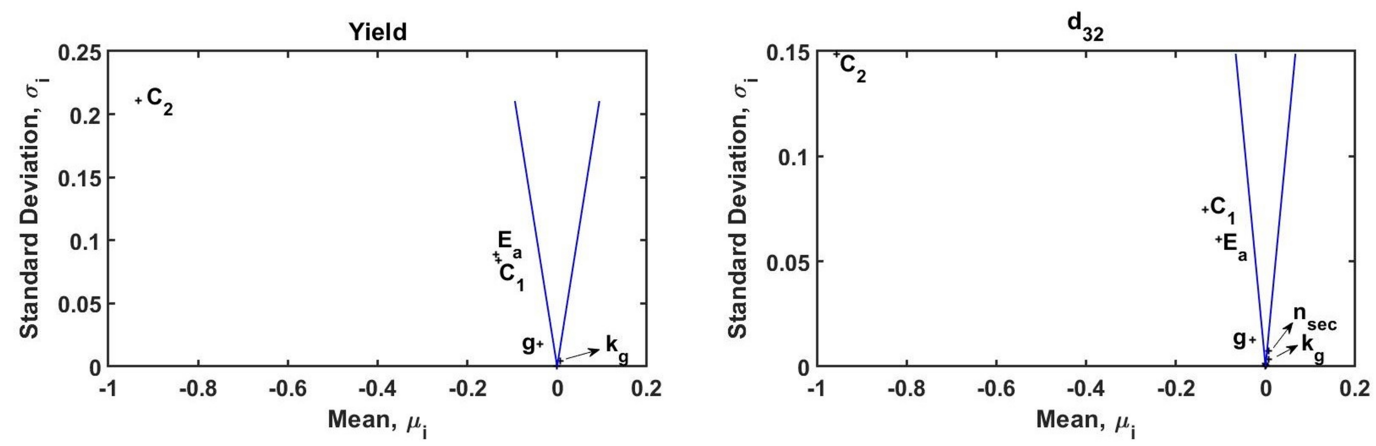

(a) Uncertainties in kinetic parameters.
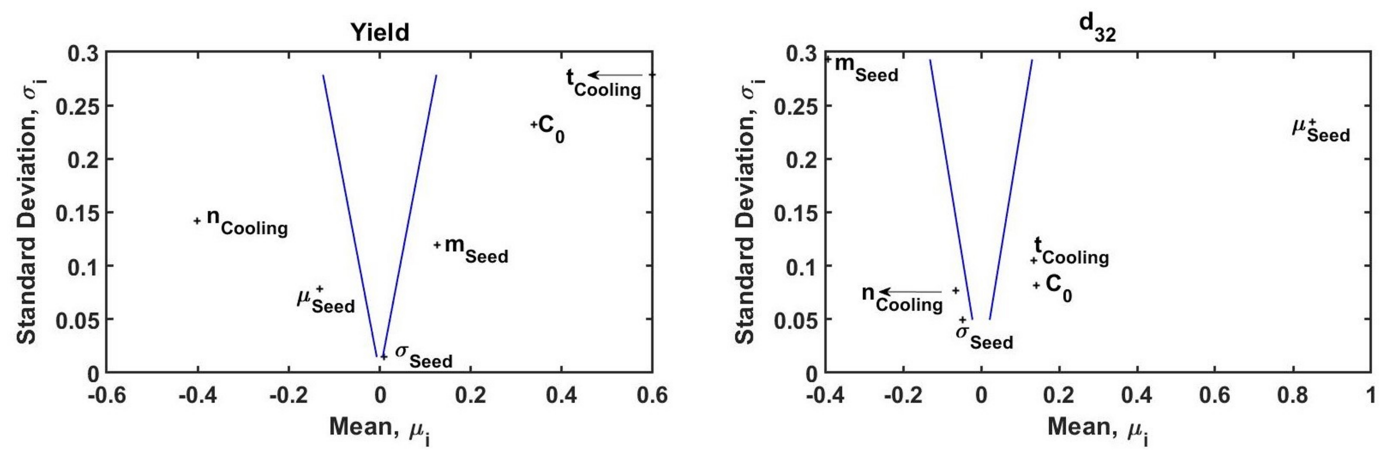

(b) Variations in operation design space.

Figure 8: Comparison of Morris screening results for kinetic parameters and operation parameters for two output of interests; process yield and final mean crystal size. Parameters that have a mean value very close to zero are not shown. 


\subsection{Case Study 2 - Antisolvent Crystallization}

\subsubsection{Uncertainty Analysis}

Operation parameters of an antisolvent crystallization process are of critical importance for optimization of the process performance and assessment of the process risks of not achieving production target. Crystallization temperature, $T_{0}$, initial supersaturation, $S_{0}$, feeding time, $t_{\text {feed }}$ (or antisolvent addition rate, $F_{a}$ ), seed specifications (mass, $m_{\text {seed }}$, mean, $\mu_{\text {seed }}$ and standard deviation, $\left.\sigma_{\text {seed }}\right)$ and initial antisolvent weight fraction, $w_{A 0}$ are chosen as the operation parameters of interest and the operation space for those parameters are given in Table 2. 600 random samples from input space were created based on LHS technique. A uniform distribution is assumed during the sampling except for antisolvent feed time. Sampling matrix can be found in Supplementary Material.

Using 600 samples, 600 Monte Carlo simulations are performed and outputs are plotted in Fig. 9. Discontinuities in solute concentration profile and mean crystal size evaluation in Fig. 9 are due the fact that antisolvent feeding time is varied as three discrete values between $1000-4000 \mathrm{~s}$, which is defined as the boundaries of the operation design space in previously Table 2. The results show that the variations in input operation space lead to high uncertainties in the process performance and consequently the product specification, especially final mean crystal size, $d_{32}$. Average $d_{32}$ of Monte Carlo simulations at the end of the antisolvent crystallization due to variations in the operation design space is calculated as $263.3 \pm 104.7 \mu \mathrm{m}$. Finally, a high average final process yield with low standard deviation is obtained as $93.7 \pm 8.2 \%$ from Monte Carlo simulations. 

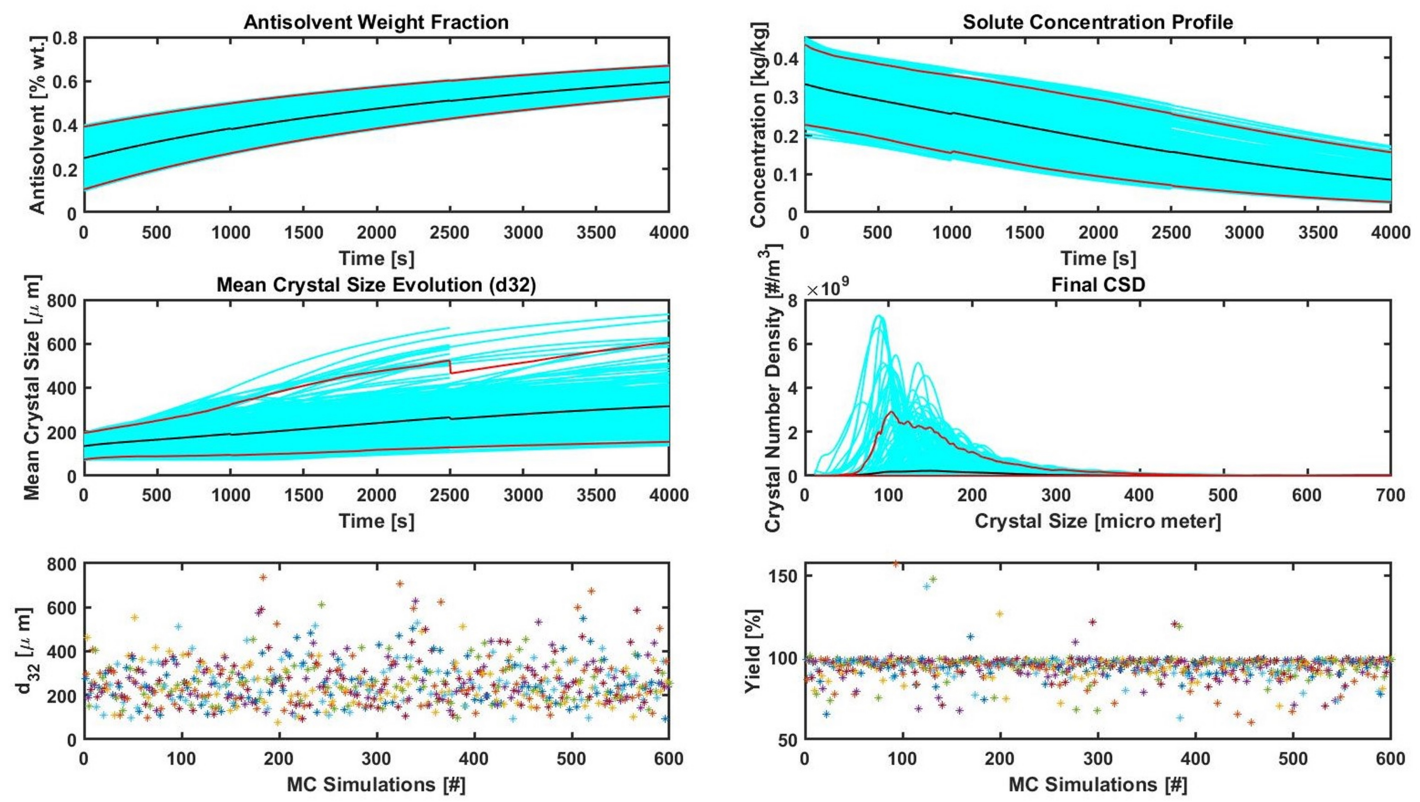

Figure 9: $600 \mathrm{MC}$ simulations: Total output uncertainty of antisolvent crystallization due to variations in input operation design space.

\subsubsection{Sensitivity Analysis}

In order to address the individual contribution of operation parameters to process output variance, a sensitivity analysis is performed based on PCE based Sobol's indices. Yield and final mean crystal size are considered as the performance criteria in the sensitivity analysis. Calculated Sobol's sensitivity indices are shown in Table 4. Calculated indices show that the antisolvent crystallization yield percentage is strongly affected by antisolvent feed time showing similarity to the cooling time in the batch cooling crystallization. On the other hand, seed mean and mass are the most sensitive parameters in determining the final mean crystal size. Consequently, focus should be given primarily to antisolvent feed time in order to optimize process yield, while crystallization temperature between specified design range has almost no effect on the final yield. Seed specifications should be focus of optimization if the purpose is to optimize final mean crystal size. 
Table 4: Ranking of the operation parameters based on PCE-based Sobol's indices. Output performance criteria are final yield percentage and final mean crystal size $\left(d_{32}\right)$.

\begin{tabular}{llcccccc}
\hline & \multicolumn{3}{c}{ Yield\% } & \multicolumn{5}{c}{ Final mean crystal size, } & $d_{32}$ \\
\hline Rank & Parameter & $S_{i}$ & $S_{T i}$ & Rank & Parameter & $S_{i}$ & $S_{T i}$ \\
\hline 1 & $t_{\text {feed }}\left(F_{a}\right)$ & 0.9914 & 0.9954 & 1 & $\mu_{\text {Seed }}$ & 0.5854 & 0.5854 \\
2 & $w_{a 0}$ & 0.0005 & 0.0045 & 2 & $m_{\text {Seed }}$ & 0.3085 & 0.3179 \\
3 & $m_{\text {seed }}$ & 0.0023 & 0.0033 & 3 & $w_{A 0}$ & 0.0662 & 0.0662 \\
4 & $\mu_{\text {seed }}$ & 0.0009 & 0.0013 & 4 & $t_{\text {feed }}\left(F_{a}\right)$ & 0.0305 & 0.0399 \\
5 & $S_{0}$ & 0.0001 & 0.0012 & & $S_{0}$ & 0 & 0 \\
6 & $T_{0}$ & 0.0000 & 0.0004 & & $T_{0}$ & 0 & 0 \\
7 & $\sigma_{\text {seed }}$ & 0 & 0.0002 & & $\sigma_{\text {seed }}$ & 0 & 0 \\
\hline
\end{tabular}

\subsection{Process Risk Quantification}

\subsubsection{Batch Cooling Crystallization}

Target crystal product quality of the batch cooling crystallization process is defined in term of yield percentage, Yield $_{\%}$ and average final crystal size diameter, $d_{32}$, and specifications are given as follow:

$$
\begin{gathered}
\text { Yield }_{\%}=\frac{\text { Yield }_{\text {Actual }}}{\text { Yield }_{\text {Theoretical }}} \cdot 100 \geq 60 \% \\
d_{32} \geq 140 \mu \mathrm{m}
\end{gathered}
$$

The undesired event is characterized as achieving a yield and final mean crystal size less than the target and the risks based on these scenarios are quantified separately. The histogram of the predicted probability distribution of Yield $\%$ and $d_{32}$ obtained from 350 Monte Carlo simulations are shown in Fig. 10 (on the left side), respectively. Cumulative density functions of the probability distributions are calculated for corresponding product quality measures and and shown in Fig. 10 as well (on the right side). The dashed line on the graph shows the target and the shadowed region shows the probability of failing target. 

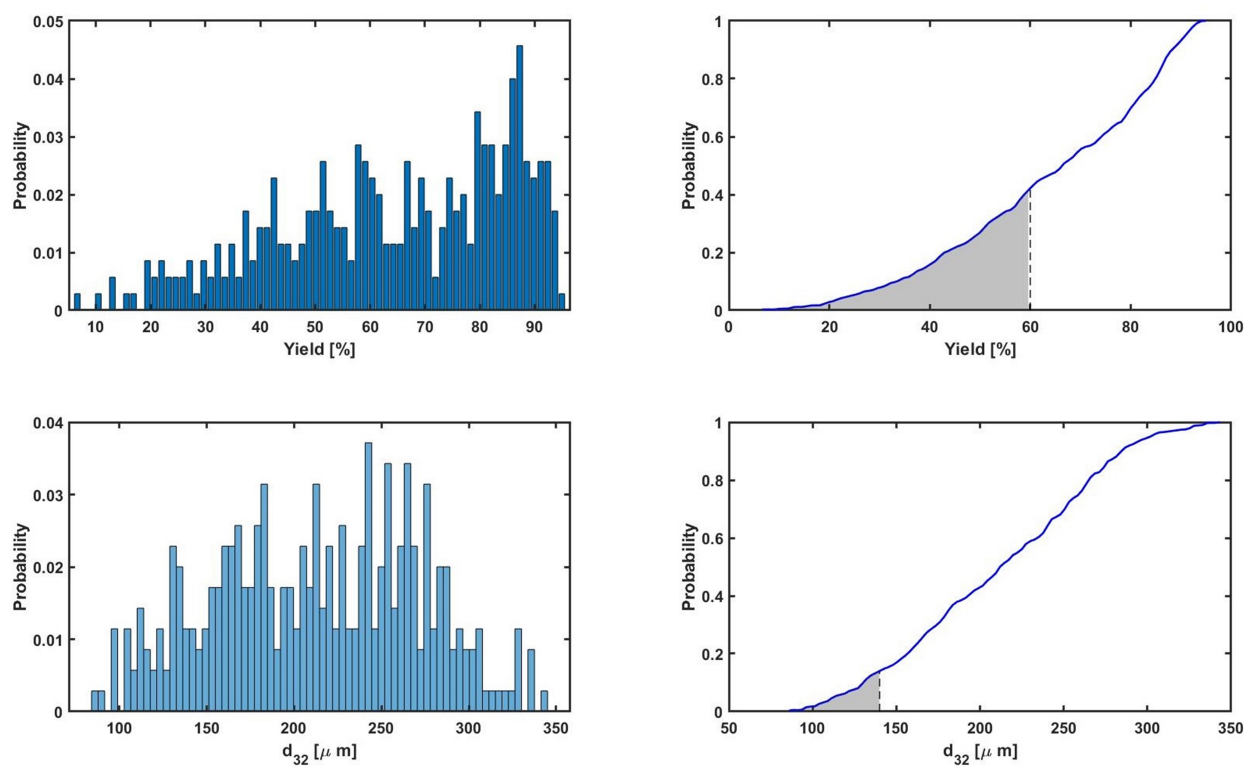

Figure 10: Histogram of of the predicted probability distribution of $Y$ ield $\%$ and $d_{32}$ obtained from 350 Monte Carlo simulations (on the left side). Cumulative density functions of the predicted values (on the right side).

In the given operation conditions of the batch cooling crystallization process, the probability of not achieving target yield is calculated as $41.5 \%$ and average final crystal size diameter is as $14.0 \%$. This means 145 and 49 simulations out of 350 simulations fail to achieve target $Y$ ield $\%$ and $d_{32}$ specifications, respectively. If both yield and final mean crystal diameter specifications are required simultaneously as a success criteria of the process, then the probability of failing target will increase up to $46.6 \%$ instead of $14.0 \%$.

The consequence of failing target Yield $_{\%}$ is the product loss. If the process risk is assessed based on the Yield $_{\%}$ specification, the overall risk is calculated as $7.1 \%$ product yield loss. On the other hand, the batch will be thrown away or wasted in consequence of failing target $d_{32}$ that will have an economic impact (cost of product and solvent) as well as an environmental impact (solvent waste). A pilot scale batch of $218 \mathrm{~L}$ crystallization contains approximately $46.8 \mathrm{~kg}$ paracetamol and $172.0 \mathrm{~kg}$ ethanol (assuming $0.272 \mathrm{~kg} / \mathrm{kg}$ API concentration). When the process is scaled up to $10,000 \mathrm{~L}$ bearing in mind the industrial production, approximately $2146.1 \mathrm{~kg}$ paracetamol and $7890.0 \mathrm{~kg}$ ethanol are wasted when only one batch is thrown away. This will cost an average 12,300 $\$$ per batch [55, 56]. On the other hand, the risk of failing product specification of $d_{32}$ under given operation design 
space of the batch cooling crystallization is calculated as app. 1,700 $\$$ per batch, when the crystallized API is paracetamol. However, it is worth to emphasize that paracetamol is one of the cheapest APIs in the drug market. As, the prices of APIs changes drastically that will change the risks of the process as well [55]. For example, under the same conditions the process risk calculated based on a drug that costs $500 \$ / \mathrm{kg}$ would be app. 151,700 $\$$ per batch considering a high value API. The risk rises up to 504,940 \$ per batch, if both target yield and final mean crystal size specifications are required as a success criteria of the process. The second consequence of environmental impact due to waste of solvent, which is ethanol in given case, can be evaluated based on different measures including some of the midpoint characterization factors (CFm) of Life Cycle Assessment (LCA) [57, 58. Calculated environmental risks due to waste of solvent ethanol per batch is give in the following Table 5 :

Table 5: Environmental risk calculations (per batch of cooling crystallization) based on various CFm of LCA due to wasting ethanol solvent.

\begin{tabular}{llll}
\hline Impact category - CF & CFm & Unit & Risk \\
\hline Global warming potential - GWP & 1.19 & $\mathrm{~kg} \mathrm{CO}$ to air & $1.32 \cdot 10^{3}$ \\
Fresh water ecotoxicity - ETPfw & $2.24 \cdot 10^{-6}$ & $1,4-\mathrm{DCP} \mathrm{eq} . \mathrm{em} . \mathrm{to} \mathrm{fw}$ & $2.47 \cdot 10^{-3}$ \\
Ecosystem ozone formation pot. - EOFP & 0.20 & $\mathrm{~kg} \mathrm{NO}-\mathrm{eq} / \mathrm{kg}$ & $2.21 \cdot 10^{2}$ \\
\hline
\end{tabular}

It is worth to mention also, that impurities are not incorporated into the developed model. However, it is well known that the presence of even small amount of impurities can have a substantial impact on the crystallization kinetics in reality such a suppressing nucleation or both suppressing or enhancing growth. Impurities can also change the growth morphology of the crystals [59, 60]. Changes in crystallization kinetic habits will affect the process output as well as the related uncertainty, and hereby the risk.

\subsubsection{Antisolvent crystallization}

Similar to previous case study, target crystal product quality of the antisolvent crystallization process is defined in term of yield percentage, Yield $\% \geq 90 \%$ and final mean crystal size, $d_{32} \geq 300 \mu \mathrm{m}$. The undesired event is defined as achieving a yield and final mean 
crystal size less than the target. The histogram of the predicted probability distribution of Yield $\%$ and $d_{32}$ obtained from 600 Monte Carlo simulations as well as cumulative density functions of the probability distributions are calculated for corresponding process/product quality measures and the graphs can be found in Supplementary Material. In the specified operation design space of the antisolvent process, the probability of not achieving target yield is calculated as $18.2 \%$ and average final crystal size is as $68.7 \%$. This means 109 and 412 simulations out of 600 simulations fail to achieve target $Y$ ield $\%$ and $d_{32}$ specifications, respectively. The probability of failing target will increase up to $71.8 \%$, if both yield and final mean crystal diameter specifications are required simultaneously as a success criteria of the process. Similar consequences are assumed in the case of failing target process and product specifications. Therefore, when the process risk is assessed based on the Yield\% criterion, the overall risk is calculated as $1.5 \%$ product yield loss. On the other hand, when the batch is thrown away or wasted in consequence of failing target $d_{32}$, approximately 2981.7 $\mathrm{kg}$ aspirin, $3623.9 \mathrm{~kg}$ ethanol and $5412.8 \mathrm{~kg}$ water are wasted per batch. The economic consequence is therefore 13,630 $\$$ per batch and the economic risk is calculated as app. 9,400 $\$$ per batch. The risk increases up to $14,250 \$$ per batch, if both target specifications are required at the same time. Calculated environmental risks due to waste of solvent ethanol per batch is give in the following Table 6 (for the second criteria only):

Table 6: Environmental risk calculations (per batch of antisolvent crystallization) based on various CFm of LCA due to wasting ethanol solvent.

\begin{tabular}{llll}
\hline Impact category - CF & CFm & Unit & Risk \\
\hline Global warming potential - GWP & 1.19 & $k g C O_{2}$ to air & $2.96 \cdot 10^{3}$ \\
Fresh water ecotoxicity - ETPfw & $2.24 \cdot 10^{-6}$ & $1,4-D C P$ eq. em. to fw & $5.58 \cdot 10^{-3}$ \\
Ecosystem ozone formation pot. - EOFP & 0.20 & $k g N O_{x}-e q / k g$ & $4.98 \cdot 10^{2}$ \\
\hline
\end{tabular}

These process risk calculations assist in framing the process scale-up and development studies and help better understand and quantify both economic and environmental consequences/impact of process failure for a given design strategy. 


\section{Conclusions}

An in silico tool for a pharmaceutical crystallization process, which serves the purpose of supporting an efficient and effective process development and optimization studies, was developed. A batch cooling crystallization of paracetamol and an antisolvent crystallization of acetylsalicylic acid were demonstrated as two case studies. To this end, the pilot scale crystallizer at $218 \mathrm{~L}$ scale was modelled based on a multi-compartment approach that couples the mixing characteristics obtained by CFD simulations and crystallization kinetics. Crystallization kinetic model parameters and the operation design parameters were considered as a source of uncertainty and variations. Monte Carlo simulation method showed huge uncertainties in model outputs due to input parameter uncertainties and variations. The global sensitivity analysis (Morris screening and variance decomposition) was applied in order to quantify individual contributions of input parameters on the output uncertainties. Sensitivity analysis results revealed that second coefficient of the solubility term has the highest effect on the process performance in terms of process yield and final mean crystal size. Therefore, care should be take to design better experiments to determine this parameter more accurately. Besides, among operation design parameters, cooling/antisolvent feed time has the highest influence on the process yield, while seed mean has the highest effect on final mean crystal size. Therefore, more efforts should be given to those significant parameters in order to optimize the related outcome of interest and reduce uncertainties, while non-influential parameters can be excluded from the optimization studies. Finally, the risk of failing process/product target due to variations in the operation design space was quantified.

This promising study shows that global uncertainty and sensitivity analysis coupled with the quantification of process risk assessment is a powerful tool and should be interest to those participating in effective and efficient crystallization process development. Developed in silico tool provides a fast and cost-efficient platform for investigating design spaces, testing the feasibility of different strategies, assessing the impact of uncertainties, identifying the determinants of the process, and quantifying the process risk. Some experimental efforts 
are still required to collect data in order to determine accurately the model parameters $(e . g$. solubility, crystallization kinetics). These data are not usually available at the start of a round development for a new API. However, these efforts can be minimized by adopting hybrid or systematic approaches that yield targeted experimental design with minimum numbers (e.g. for solubility [61] and for crystallization kinetics [62]).

\section{Acknowledgement}

We would like to thank the Danish Council for Independent Research (DFF) for financing the project with grant ID: DFF-6111600077B.

\section{Appendix A. Supplementary Material}

Supplementary material associated with this article can be found in the online version.

\section{References}

\section{References}

[1] J. Wang, F. Li, R. Lakerveld, Process intensification for pharmaceutical crystallization, Chemical Engineering \& Processing: Process Intensification 127 (2018) 111-126.

[2] J. Chen, B. Sarma, J. M. Evans, A. S. Myerson, Pharmaceutical crystallization, Crystal Growth \& Design 11 (2011) 887-895.

[3] Z. Gao, S. Rohani, J. Gong, J. Wang, Recent developments in the crystallization process: Towards the pharmaceutical industry, Engineering 3(3) (2017) 343-353.

[4] Z. Q. Yu, J. W. Chew, P. Chow, R. B. H. Tan, Recent advanced in crystallization control an industrial perspective, Chemical Engineering Research and Design 85(A7) (2007) 893-905.

[5] Z. K. Nagy, M. Fujiwara, R. D. Braatz, Modelling and control of combined cooling and antisolvent crystallization process, Journal of Process Control 18 (2008) 856-864. 
[6] P. McKenzie, S. Kiang, J. Tom, A. E. Rubin, M. Futran, Can pharmaceutical process development become high tech?, AIChe Journal 52 (2006) 3990-3994.

[7] U. S. Food and Drug Administration, Pharmaceutical CGMPs for the $21^{\text {st }}$ century - A risk-based approach (2004).

[8] U. S. Food and Drug Administration, PAT - A framework for innovative pharmaceutical development, manufacturing, and quality assurance (2004).

[9] K. L. Lacki, J. Joseph, K. O. Eriksson, Biopharmaceutical Processing Development, Design, and Implementation of Manufacturing Processes, Elsevier, Oxford, 2018, Ch. Downstream Process Design, Scale-Up Principles, and Process Modeling.

[10] Z. K. Nagy, R. D. Braatz, Distributional uncertainty analysis using power series and polynomial chaos expansions, Journal of Process Control 17 (2007) 229-240.

[11] T. Vetter, C. L. Burcham, M. F. Doherty, Designing robust crystallization processes in the presence of parameter uncertainty using attainable regions, Industrial \& Engineering Chemistry Research 54 (2015) 10350-10363.

[12] E. Bolaños-Reynoso, K. B. Sánchez-Sánchez, G. R. Urrea-García, L. Ricardez-Sandoval, Dynamic modeling and optimization of batch crystallization of sugar cane under uncertainty, Industrial \& Engineering Chemistry Research 53 (2014) 13180-13194.

[13] F. C. C. Montes, K. Gernaey, G. Sin, Dynamic plantwide modeling, uncertainty, and sensitivity analysis of a pharmaceutical upstream synthesis: Ibuprofen case study, Ind. Eng. Chem. Res. 57(30) (2018) 10026-10037.

[14] D. Fysikopoulos, B. Benyahi, A. Borsos, Z. K. Nagy, C. D. Rielly, A framework for model reliability and estimability analysis of crystallization processes with multi-impurity multi-dimensional population balance models, Computers and Chemical Engineering $122(2019) 275-292$.

[15] J. A. Selekman, D. Roberts, V. Rosso, J. Qiu, J. Nolfo, Q. Gao, J. Janey, Development of a hihgly automated workflow for investigating polymorphism and assessing risk of 
forming undesired crystal forms within a crystallization design space, Organic Process Research \& Development 20 (2016) 70-75.

[16] Z. Q. Yu, P. S. Chow, R. B. H. Tan, Design space for polymorphic co-crystallization: Incorporating process model uncertainty and operational variability, Crystal Growth \& Design 14 (2014) 3949-3957.

[17] M. Öner, C. Bach, T. Tajsoleiman, G. S. Molla, M. F. Freitag, S. M. Stocks, J. Abildskov, U. Krühne, G. Sin, Scale-up modeling of a pharmaceutical crystallization process via compartmentalization approach, Computer Aided Chemical Engineering 44 (2018) 181-186.

[18] C. Fernandes, Effect of nature of the solvent on the crystallization of paracetamol, accepted for 14th International Symposium on Industrial Crystallization (ISIC 14) (1999) September.

[19] N. A. Mitchell, Numerical modeling of cooling crystallisation: Process kinetics to optimization, Ph.D. thesis, Department of Mechanical, Aeronautical \& Biomedical Engineering, University of Limerick (2012).

[20] C. Lindenberg, M. Krättli, J. Cornel, M. Mazotti, Design and optimization of a combined cooling/antisolvent crystallization process, Crystal Growth \& Design 9 (2009) 1124-1136.

[21] D. Ramkrishna, Population Balances: Theory and Applications to Particulate Systems in Engineering, Academic Press, 1th ed. edition, San Diego, 2000.

[22] P. Marchal, R. David, J. P. Klein, J. Villermaux, Crystallization and precipitation engineering-I. an efficient method for solving population balance in crystallization with agglomeration, Chemical Engineering Science 43 (1988) 59-67.

[23] F. Puel, G. Fvotte, J. P. Klein, Simulation and analysis of industrial crystallization processes through multidimensional population balance equations. Part 1: A resolution 
algorithm based on the method of classes, Chemical Engineering Science 58 (2003) $3715-3727$.

[24] M. Öner, S. M. Stocks, J. Abildskov, G. Sin, Scale-up modeling of a pharmaceutical antisolvent crystallization via a hybrid method of computational fluid dynamics and compartmental modeling, Computer Aided Chemical Engineering 46 (2019) 709-714.

[25] H. K. Larsson, Modelling of mass transfer phenomena in chemical and biochemical reactor systems using computational fluid dynamics, Ph.D. thesis, Department of Chemical and Biochemical Engineering, Technical University of Denmark (2015).

[26] G. J. Wells, W. H. Ray, Methodology for modeling detailed imperfect mixing effects in complex reactors, AIChE Journal 51 (2005) 1508-1520.

[27] E. K. Nauha, Z. Kálal, J. M. Ali, V. Alopaeus, Compartmental modeling of large stirred tank bioreactors with high gas volume fractions, Chemical Engineering Journal 334 (2018) 2319-2334.

[28] E. Kougoulos, A. G. Jones, M. W. Wood-Kaczmar, A hybrid CFD compartmentalization modeling framework for the scaleup of batch cooling crystallization processes, Chem. Eng. Comm. 193 (2006) 1008-1023.

[29] A. Nørregaard, C. Bach, U. Krühne, U. Borgbjerg, K. V. Gernaey, Hypothesis-driven compartment model for stirred bioreactors utilizing computational fluid dynamics and multiple pH sensors, Chemical Engineering Journal 356 (2019) 161-169.

[30] E. K. Nauha, V. Alopaeus, Modeling method for combining fluid dynamics and algal growth in a bubble photobioreactor, Chemical Engineering Journal 229 (2013) 559-568.

[31] A. Saltelli, Sensitivity analysis for importance assessment, Risk Analysis 3 (2002) 579590.

[32] A. Saltelli, K. Aleksankina, W. Becker, P. Fennell, F. Ferreti, N. Holst, S. Li, Q. Wu, Why so many published sensitivity analyses are false. A systematic review of sensitivity analysis practices (2017). 
[33] J. C. Helton, F. J. Davis, Latin hypercube sampling and the propagation of uncertainty in analyses of complex systems, Reliability Engineering \& System Safety 81 (2003) 2369.

[34] G. Sin, K. V. Gernaey, Data handling and parameter estimation, in: M. C. M. van Loosdrect, P.H. Nielsen, C. M. Lopez-Vazquez, D. Brdjanovic (Eds.), Experimental Methods in Wastewater Treatment, IWA Publishing, London, 2016, pp. 201-234.

[35] M. D. McKay, R. J. Beckman, W. J. Conover, A comparison of three methods for selecting values of input variables in the analysis of output from a computer code, Technometrics 21 (1979) 239-245.

[36] M. D. Morris, Factorial sampling plans for preliminary computational experiments, Technometrics 33 (1991) 161-174.

[37] G. Sin, K. V. Gernaey, A. E. Lantz, Good modeling practice for PAT applications: Propagation of input uncertainty and sensitivity analysis, Biotechnol. Prog. 25 (2009) $1043-1053$.

[38] J. D. Herman, J. B. Kollat, P. M. Reed, T. Wagener, Technical note: Method of Morris effectively reduces the computational demands of global sensitivity analysis for distributed watershed models, Hydrology and Earth Systems Sciences 17 (2013) 28932903.

[39] A. Zubov, G. Sin, Multiscale modeling of poly(lactic acid) production: From reaction conditions to rheology of polymer melt, Chemical Engineering Journal 336 (2018) 361375.

[40] A. F. Campolongo, A. Saltelli, Sensitivity analysis of an environmental model: An application of different analysis methods, Reliability Engineering and System Safety 57 (1997) 49-69.

[41] A. F. Campolongo, J. Cariboni, A. Saltelli, An effective screening design for sensitivity analysis, Environ. Model Soft. 22 (2007) 1509-1518. 
[42] A. Saltelli, P. Annoni, I. Azzini, F. Campolongo, M. Ratto, S. Tarantola, Variance based sensitivity analysis of model output. Design and estimator for the total sensitivity index, Computer Physics Communications 181 (2010) 259-270.

[43] I. Sobol, Sensitivity estimates for nonlinear mathematical models, Matematicheskoe Modelirovanie 2 (1990) 112-118.

[44] I. Sobol, Sensitivity estimates for nonlinear mathematical models, Mathematical Modeling \& Computational Experiment 1 (1993) 407-414.

[45] T. Homma, A. Saltelli, Importance measures in global sensitivity analysis of nonlinear models, Reliability Engineering and System Safety 52 (1996) 1-17.

[46] R. Al, C. Behera, A. Zubov, K. V. Gernaey, G. Sin, Meta-modeling based efficient global sensitivity analysis for wastewater treatment plants - An application to BSM2 model, Computers and Chemical Engineers 127 (2019) 233-246.

[47] B. Sudret, Global sensitivity analysis using polynomial chaos expansions, Reliab. Eng. Syst. Saf. 93 (2008) 964-979.

[48] S. Marelli, B. Sudret, Uqlab: A framework for uncertainty quantification in Matlab, Proc. 2nd Int. Conf. on Vulnerability, Risk Analysis and Management (ICVRAM2014) (2014) 2554-2563.

[49] A. Marrel, B. Iooss, B. Laurent, O. Roustant, Calculation of Sobol indices for the Gaussian process metamodel, Reliab. Eng. Syst. Saf. 94 (2009) 742-751.

[50] S. Li, B. Yang, F. Qi, Accelerate global sensitivity analysis using artificial neural network algorithm: Case studies for combustion kinetic model, Combust. Flame 168 (2016) $53-64$.

[51] I. T. Cameron, R. Raman (Eds.), Process Systems Risk Management, Elsevier Inc., San Diego, 2005.

[52] R. Spann, K. V. Gernaey, G. Sin, A compartment model for risk-based monitoring of lactic acid bacteria cultivations, Biochemical Engineering Journal 151 (2019) 107293. 
[53] R. Brun, M. Kühni, H. Siegrist, W. Gujer, P. Reichert, Practical identifiability of ASM2d parameters - systematic selection and tuning of parameter subsets, Water Research 36 (2002) 4113-4127.

[54] J. Worlitschek, M. Mazzotti, Model-based optimization of particle size distribution in batch-cooling crystallization of paracetamol, Crystal Growth \& Design 4 (2004) 891903.

[55] A. M. Hill, M. J. Barber, D. Gotham, Estimated costs of production and potential prices for the WHO essential medicines list, BMJ Global Health 3 (2017) e000571.

[56] Ethanol price commodity, url = https://markets.businessinsider.com/ commodities/ethanol-price, note $=$ Accessed: 2019-09-24.

[57] A. Amelio, G. Genduso, S. Vreysen, P. Luis, B. V. der Bruggen, Guidelines based on life cycle assessment for solvent selection during the process design and evaluation of treatment alternatives, Green Chemistry 16 (2014) 3045-3063.

[58] M. A. J. Huijbregts, Z. J. N. Steinmann, P. M. F. Elshout, G. Stam, F. Verones, M. D. M. Vieira, A. Hollander, M. Zijp, R. van Zelm, ReCiPe 2016 A harmonized life cycle impact assessment method at midpoint and endpoint level - Report I: Characterization, National Institute for Public Health and the Environment.

[59] K. V. R. Prasad, R. I. Ristic, D. B. Sheen, J. N. Sherwood, Crystallization of paracetamol from solution in the presenceand absence of impurity, International Journal of Pharmaceutics 215 (2001) 29-44.

[60] A. M. A. Borsos, Z. K. Nagy, Multi-impurity adsorption model for modeling crystal purity and shape evolution during crystallization process in impure media, Crystal Growth \& Design 16 (2016) 555-568.

[61] G. S. Molla, M. F. Freitag, S. M. Stocks, K. T. Nielsen, G. Sin, Solubility prediction of different forms of pharmaceuticals in single and mixed solvents using symmetric 
electrolyte nonrandom two-liquid segment activity coefficient model, Ind. Eng. Chem. Res 58(10) (2019) 4267-4276.

[62] A. Eren, B. Szilágyi, J. Quon, M. Furuta, C. Papageorgiou, Z. K. Nagy, Development of a model-based quality-by-control framework for crystallization design, Computer Aided Chemical Engineering 46 (2019) 319-324. 